duplicará en los próximos 15 años y que ascenderá a 10 millones de personas en 2020 .

La Encuesta Mundial sobre Tabaquismo en Jóvenes (EMTJ) es un proyecto conjunto de la Organización Mundial de la Salud (OMS), los Centros para el Control y la Prevención de Enfermedades de los Estados Unidos de América (CDC), la Asociación Canadiense de Salud Pública (CPHA) y la mayoría de los Estados Miembros de la Organización Mundial de la Salud (OMS). Este proyecto se basa en numerosas encuestas transversales realizadas con muestras independientes y representativas de estudiantes de 13 a 15 años que fueron entrevistados en las escuelas escogidas. Entre 1999 y 2005 se realizaron 750000 encuestas en casi 10000 escuelas de 131 países, la franja de Gaza y Cisjordania. Treinta y siete de los países estaban en las Américas. La tasa de respuesta fue de $44,2 \%$ a $97,1 \%$ (de $50,0 \%$ a $100 \%$ entre las escuelas y de $58,0 \%$ a $99,7 \%$ entre los estudiantes).

Los resultados de este trabajo y de investigaciones anteriores realizadas en el marco del proyecto EMTJ demostraron que la diferencia en la frecuencia del tabaquismo entre niños y niñas es menor que la observada entre hombres y mujeres. Además, en todas las regiones, salvo por América y Europa, se observó que los estudiantes consumían tabaco en forma de productos distintos del cigarrillo con la misma o más frecuencia con la que consumían cigarrillos. Cerca de $20 \%$ de los estudiantes encuestados que nunca habían fumado manifestaron que era posible que empezaran a fumar el año siguiente. La exposición de los estudiantes al humo de cigarrillos ajenos era frecuente, tanto en el hogar (30\%) como en lugares públicos (45\%).

Este estudio posee varias limitaciones. Una de ellas es que solo refleja la situación de los adolescentes de 13 a 15 años que asisten a la escuela, por lo que sus resultados no son representativos de todos los jóvenes de esa edad. Además, se basa en autoinformes de los estudiantes que podrían no reflejar la realidad con exactitud.

Estos resultados respaldan la sospecha de que la incidencia de enfermedades crónicas y la mortalidad relacionadas con el tabaquismo se mantendrá elevada en los próximos años. Se deben redoblar los esfuerzos para reducir el consumo de tabaco, en particular para evitar el inicio del hábito de fumar y conseguir su abandono en la población juvenil. La elevada exposición de los jóvenes al humo de los cigarrillos de otros fumadores confirma la necesidad de establecer políticas más enérgicas y eficaces que protejan a los jóvenes que no fuman. (Warren CW et al. Patterns of global tobacco use in young people and implications for future chronic disease burden in adults. Lancet. 2006;367(9512):749-53.)

\section{Papel de las comadronas en las comunidades cakchiqueles de Guatemala}

En el departamento de Sololá, en Guatemala, cuya población es predominantemente indígena, las mujeres prefieren dar a luz en su casa bajo el cuidado de comadronas antes que hacerlo en el puesto médico local. En esta zona, la mayor parte de las muertes relacionadas con el embarazo ocurren en los hogares y cuando una mujer acude al hospital, su estado suele ser muy grave por haber esperado demasiado tiempo antes de buscar ayuda especializada.

En este trabajo se analizan, mediante un enfoque etnográfico cualitativo, las razones por las cuales las mujeres cakchikeles no acuden directamente al hospital tan pronto surgen complicaciones durante su embarazo. Para ello se realizó un estudio de campo de 2 años (de septiembre de 2000 a agosto de 2001 y de septiembre de 2002 a agosto de 2003) en tres poblados de Sololá.

En esta población, el parto se basa en la relación entre la embarazada, su familia y la comadrona. Las comadronas legas y los miembros de la familia no están capacitados para indicar la remisión de una paciente a un servicio determinado. Sin embargo, las dificultades son mucho más complejas y van más allá de la imposibilidad de "adquirir la información y los conocimientos necesarios". El problema no se circunscribe a diferencias de conducta, sino a que en ese grupo étnico el parto es un suceso social a la vez que biológico. El conocimiento está anclado a un sistema social mucho más amplio.

En tales condiciones, las decisiones que se toman cuando hay una emergencia durante el parto no dependen de creencias estáticas o de determinada información, sino de negociaciones entre los participantes. Estas negociaciones no se limitan a la evaluación de la información biológica disponible, sino que están dirigidas a lograr el mayor beneficio para cada uno de los actores involucrados, es decir, para la futura madre, sus familiares y la comadrona. Por consiguiente es un error pensar que las creencias culturales son una fuerza estática que actúa para lograr determinados resultados.

Desde el punto de vista económico se comprende que el Ministerio de Salud Pública y Asistencia Social (MSPAS) de Guatemala haya adop- 
tado un sistema de remisión o derivación que trata de dirigir los escasos recursos - por ejemplo, los mejores profesionales y el equipo más avanzadoa las personas que más los necesitan. Según un estudio inicial, esta política ha logrado buenos resultados en zonas pobres de la Ciudad de Guatemala, donde las comadronas tradicionales trabajan en centros locales de maternidad bajo la supervisión de un médico y del personal de enfermería.

No obstante, un sistema basado en la derivación para responder a las emergencias obstétricas puede no tener éxito si no hay un médico que supervise el parto. A pesar de que el MSPAS ha tratado de aliviar este problema mediante el entrenamiento de comadronas cakchiqueles que atienden los partos en los hogares, no se puede esperar que estas abandonen sus costumbres y prácticas solo para cumplir con las directivas del MSPAS. Esas directivas deben adaptarse al sistema de conocimientos ya existente.

Aunque las directivas promueven las derivaciones preventivas y hacen un llamado a las mujeres a que acudan a los hospitales antes de que surjan complicaciones, no tienen en cuenta el deseo de las embarazadas de tener un parto vaginal normal en su hogar, rodeada de sus familiares. Por eso, la mayoría de las mujeres cakchiqueles preferirán esperar a que surja un problema para acudir al hospital, antes de sacrificar innecesariamente la rica experiencia de un parto en las condiciones que ellas desean. (Berry NS. Kaqchikel midwives, home births, and emergency obstetric referrals in Guatemala: contextualizing the choice to stay at home. Soc Sci Med. 2006;62(8):1958-69.)

\section{Percepciones acerca del uso de fármacos abortivos en mujeres de Colombia, Ecuador, México y Perú}

A pesar de que el aborto está restringido legalmente en casi todos los países de América Latina, los servicios dedicados a esa actividad están ampliamente disponibles, especialmente en las áreas urbanas. Los hay caros y seguros o baratos y más peligrosos. Se calcula que $29 \%$ de las mujeres en edad reproductiva se someten a abortos mediante procedimientos riesgosos, y las mujeres pobres o que viven en áreas rurales se encuentran aun en mayor desventaja. Según los códigos penales de Colombia, Ecuador, México y Perú, el aborto inducido es un crimen, salvo muy pocas excepciones. Se calcula que entre 1995 y 2000, alrededor de $28 \%$ de las muertes maternas en Colombia, $18 \%$ en Ecuador, $23 \%$ en México y $16 \%$ en Perú se debieron a abortos riesgosos. Aunque se han realizado pocos esfuerzos para mejorar el acceso a procedimientos más seguros, se han puesto en marcha programas para la atención de las complicaciones provocadas por los abortos.

Se realizaron entrevistas a profundidad a 49 mujeres entre 18 y 44 años de edad (mediana: 25 años) que habían consumido un fármaco abortivo bajo supervisón clínica en zonas rurales urbanas de Colombia, Ecuador, México y Perú, con la finalidad de conocer su percepción sobre el tema. La captación de estas mujeres para la investigación estuvo a cargo de los propios médicos que realizaron los abortos; las entrevistas se efectuaron entre octubre de 2003 y mayo de 2004.

Las participantes de los cuatro países prefirieron someterse al uso de un abortivo por ser menos doloroso, más sencillo, más natural, menos invasor y menos peligroso que el aborto quirúrgico. Por lo general lo describieron como un proceso natural de regulación menstrual. El hecho de que ofrecía mayor privacidad y era menos costoso también influyó en la decisión.

A excepción de las participantes de México, las mujeres encuestadas no estaban bien informadas acerca de la necesidad de emplear métodos anticonceptivos con regularidad, o no habían incorporado esa información adecuadamente a su forma de vida. Algunas entrevistadas afirmaron que sus parejas habían ofrecido tomar las precauciones para evitar el embarazo, pero que no lo habían cumplido.

De las 49 entrevistadas, $37(75,5 \%)$ tuvieron un aborto satisfactorio con el fármaco ingerido, mientras que $12(24,5 \%)$ recurrieron al aborto quirúrgico después del fracaso del abortivo. En total, 38 mujeres $(77,6 \%)$ usaron misoprostol —de ellas $30(78,9 \%)$ lograron un aborto satisfactorio- y $11(22,4 \%)$ usaron metotrexato con misoprostol, con lo cual obtuvieron buenos resultados en $7(63,6 \%)$ casos. Los embarazos tuvieron una duración mediana de 7 semanas.

Las mujeres que consideraban el aborto un pecado tuvieron mayores dificultades, mientras que las que comprendían los cambios que ocurrían en su organismo al abortar soportaron mejor el proceso. La participación de un médico y el apoyo de la pareja fueron factores clave para la estabilidad de las mujeres.

El uso de un abortivo goza de la aceptación de un amplio espectro de mujeres en lugares donde se prohíbe legalmente, por lo que se debe poner a su 